\title{
The effect of clinical pharmacist-led educational tool on adherence to antiepileptic drugs in pediatrics: an interventional study
}

\author{
Suha Jarad ${ }^{1}$, Amal Akour ${ }^{1}$, Wael Khreisat ${ }^{2}$, and Afrah El-Shammari² \\ ${ }^{1}$ The University of Jordan \\ ${ }^{2}$ Queen Rania Children's Hospital
}

March 8, 2021

\begin{abstract}
Background: Rate of non-adherence to antiepileptic drugs (AEDs) in children is about 33\%. Engaging clinical pharmacists in the management of patients has proved to increase adherence to medications which will improve the outcomes of treatment.

Objectives: To investigate the effect of a clinical pharmacist-led educational tool on the adherence to AEDs in pediatric patients with epilepsy. Secondary outcomes include effectiveness and safety of AEDs, satisfaction with information about AEDs provided to the caregivers, and patients quality of life (QoL).

Methods: This was an interventional study where pediatric patients were randomly assigned to the intervention $(n=41)$ or the control $(n=40)$ group. A 30-minute clinical pharmacist-led educational interview to the parent/caregiver was provided to the intervention group as add-on to standard medical care received by the control group. Outcomes were measured at baseline and after eight-week follow-up.

Results: The intervention group had an increase in mean adherence score from $6 \pm 1.09$ at baseline to $7.6 \pm 0.9$ at follow up $(P$-value $<0.001)$, while the control group had no significant change $(P$-value $>0.05)$, the difference between the two groups at follow-up was significant $(P$-value $<0.0001)$. No significant difference was observed between groups at follow up with regard to effectiveness $(P$-value $>0.05)$, and safety $(P$-value $=0.08)$. While higher satisfaction with information $(P$-value $<0.0001)$, and higher QoL $(P$-value $<0.05)$ was observed in the intervention group. There was a significant positive correlation between satisfaction and adherence $(\mathrm{r}=0.682, P$-value $<0.0001)$, adherence and QoL $(\mathrm{r}=0.323, P$-value $<0.01)$, satisfaction and QoL $(\mathrm{r}=0.298, P$-value $<0.05)$. While, satisfaction and safety, safety and QoL correlated significantly and negatively $(\mathrm{r}=-0.263$, $P$-value $<0.05$ and $\mathrm{r}=-0.782, P$-value $<0.0001$, respectively $)$
\end{abstract}

Conclusion: Clinical pharmacist-led educational tool had a positive outcome on pediatric patients with epilepsy with regard to adherence, effectiveness, safety, satisfaction with information about AEDs, and QoL.

The effect of clinical pharmacist-led educational tool on adherence to antiepileptic drugs in pediatrics: an interventional study

Suha Jarad ${ }^{1 *}$, Amal Akour ${ }^{1,2}$, Wael H Khreisat ${ }^{3}$, Afrah K Elshammari ${ }^{3}$

${ }^{1}$ Department of Clinical Pharmacy and Biopharmaceutics, School of Pharmacy, The University of Jordan, Amman, Jordan

${ }^{2}$ Department of Pharmacy, Faculty of Pharmacy, Al-Zaytoonah University of Jordan, Amman, Jordan

${ }^{3}$ Department of Pediatric Neurology, Queen Rania Children's Hospital, Royal Medical Services, Amman, Jordan

\section{* The corresponding author}

Suha Jamal Jarad

Department of Clinical Pharmacy and Biopharmaceutics, 
School of Pharmacy, The University of Jordan, Amman, 11942 Jordan

Email: suhajarad@gmail.com

Phone No. : +962 799417325

DISCLOSURE

The authors declare no conflict of interest 